\title{
The reliability and validity of Lymphedema Quality of Life Questionnaire-Arm in Turkish patients with upper limb lymphedema related with breast cancer
}

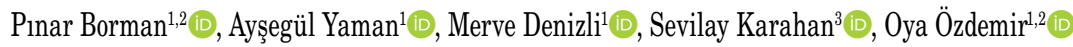 \\ 'Department of Physical Medicine and Rehabilitation, Medicine Faculty of Hacettepe University, Ankara, Turkey \\ ${ }^{2}$ University of Hacettepe Lymphedema Practice and Research Center, Ankara, Turkey \\ ${ }^{3}$ Department of Biostatistics, Medicine Faculty of Hacettepe University, Ankara, Turkey
}

Received: March 12, 2018 Accepted: June 27, 2018 Published online: July 09, 2018

\begin{abstract}
Objectives: This study aims to adapt Lymphedema Quality of Life Questionnaire-Arm (LYMQOL) into Turkish and to test its reliability and validity in Turkish patients with upper limb lymphedema related with breast cancer.

Patients and methods: Between June 2015 and November 2015, the Turkish LYMQOL-Arm was obtained using forward-backward translation method and administered to a total of 135 female patients (mean age 51.8 \pm 9.8 years; range, 31 to 82 years) with upper limb lymphedema with European Organization for Research and Treatment of Cancer-QoL Breast Cancer-specific version (EORTC QLQ-BR23) and Functional Assessment of Cancer Therapy-Breast-4 (FACT-B+4) questionnaires. A test-retest interval of seven-days was used to assess the reliability. The validation studies were carried-out by means of construct-validity using Spearman's rank correlation-coefficient. Internal consistency and test-retest-reliability were assessed using Cronbach's alpha and intra-class correlation-coefficient (ICC), respectively.

Results: 135 patients completed the questionnaire with upper limb lymphedema related with breast cancer completed the questionnaires. The mean lymphedema duration was $21.1 \pm 28.7$ (median: 6) months. Internal consistency and reliability of the Turkish LYMQOL-Arm was good with Cronbach's alpha (0.88-0.90) and test-retest ICC (0.45-0.71). External construct validity was highly confirmed by expected correlations with comparator scales, EORTCQLQ-BR23 and FACT-B+4 (p<0.01).

Conclusion: The Turkish version of the LYMQOL-Arm is a valid and reliable tool for evaluating QoL in female patients with upper limb lymphedema related with breast cancer.

Keywords: Lymphedema Quality of life Questionnaire-Arm; lymphedema; quality of life; validity; Turkish.
\end{abstract}

Breast cancer is the most frequent cancer among women in Turkey. ${ }^{[1]}$ Cancer treatments comprising resection of lymph nodes and/or radiation therapy can damage lymph drainage pathways causing accumulation of lymph fluid in the interstitial tissue of related limbs and body areas, known as secondary lymphedema. ${ }^{[2,3]}$ In recent years, more effective screening modalities and advances in therapeutic procedures have resulted in significant improvements in the survival of cancer patients. ${ }^{[4]}$ The long-term side effects and treatment-related adverse effects during the survivorship may impair the quality of life (QoL) of the patients. Lymphedema has been described as one of the most significant survivorship complications after the surgical treatment of breast cancer. ${ }^{[4-6]}$

Lymphedema may have severe consequences in terms of patients' functional and psycho-social aspects of life. Accurate information on health-related QoL outcomes among patients with lymphedema is critically needed to determine evidence-based decision making and indicate the impact of disease on survivors' lives. ${ }^{[1,5,6]}$ A number of questionnaires has been presented to assess the QoL in cancer patients

Corresponding author: Pınar Borman, MD. Hacettepe Üniversitesi Tip Fakültesi, Fiziksel Tip ve Rehabilitasyon Anabilim Dalı, 06100 Sihhiye, Ankara, Turkey. e-mail: pinarborman@gmail.com

Cite this article as:

Borman P, Yaman A, Denizli M, Karahan S, Özdemir 0. The reliability and validity of Lymphedema Quality of Life Questionnaire-Arm in Turkish patients with upper limb lymphedema related with breast cancer. Turk J Phys Med Rehab 2018;64(3):205-212. 
with lymphedema. Generic instruments comprising; European Organization for Research and Treatment of Cancer QoL Breast Cancer (EORTC-QLQ-30), its specific version for breast cancer (EORTC-QLQ-BR23), the World Health Organization Quality of Life (WHOQOL)-BREF, Functional Assessment of Cancer Therapy-Breast (FACT-B) as well as Short Form-36 (SF-36) or Nottingham Health Profile (NHP) have been used in previous studies. ${ }^{[7-10]}$ In recent years, there has been more interest in developing lymphedema-specific QoL instruments. Due to the specific symptoms and difficulties of the patients with lymphedema, it is important to use a questionnaire specifically developed for patients suffering from this chronic condition. However, few are developed to measure specific QoL in patients with lymphedema. The Upper Limb Lymphedema-27 (ULL-27), ${ }^{[11]}$ Lymphedema Quality of Life Inventory (LyQLI), ${ }^{[12]}$ Lymphedema Functioning, Disability and Health Questionnaire (Lymph-ICF), ${ }^{[13]}$ and Lymphedema Life Impact Scale (LLIS) ${ }^{[14]}$ were specifically designed and validated for assessing health related-QoL in patients with upper limb lymphedema. However, we are impressed with the short and simple questionnaire named Quality of Life measure for Limb Lymphedema (LYMQOL) developed by Keeley et al. ${ }^{[15]}$ It consists of two questionnaires: for upper and lower lymphedema LYMQOL-Arm and LYMQOL-Leg. We are surprised to find very little published translated versions in the literature.

The LYMQOL is a disease specific patient reported outcome measure developed in the United Kingdom (UK), consisting of 28 perceived items. The Turkish language is spoken by nearly 85 million individuals worldwide. Previously several generic questionnaires comprising EORTC-QLQ-30, its specific version for breast cancer-EORTC-QLQ-BR23, ${ }^{[7]}$ SF-36, ${ }^{[16]}$ and FACTB- $4^{[17]}$ have been translated to Turkish and validated for breast cancer patients. However, no specific instrument for lymphedema has been tested for validity and reliability in Turkey. In this study, we aimed to translate and validate the LYMQOL-Arm for Turkish breast cancer patients with lymphedema.

\section{PATIENTS AND METHODS}

In this study, we conducted a descriptive and methodological study for validation of the LYMQOLArm among Turkish patients with breast cancerrelated lymphedema (BCRL). The study was conducted at Lymphedema Unit, Physical Medicine and Rehabilitation Department of a university hospital, between June 2015 and November 2015. A total of
135 female patients (mean age 51.8 \pm 9.8 years; range, 31 to 82 years) were included. The study was approved by the Clinical Research Ethics Committee of the University School of Medicine. A written informed consent was obtained from each patient. The study was conducted in accordance with the principles of the Declaration of Helsinki.

The BCRL patients with having mental incapacity, having psychiatric diagnoses requiring medications, patients with other types of cancer, those younger than 18 years and older than 65 years were not included. Patients were excluded, if they were illiterate or involved in intensive lymphedema treatment during the test period. All patients completed their chemotherapy and radiotherapy sessions and did not have active malignancy. The diagnosis of BCRL was verified by bilateral volumetric measurements depending on the circumferential measurements. ${ }^{[18]}$ Circumferential measurements were performed by a standard $1 \mathrm{~cm}$ retractable tape at $4 \mathrm{~cm}$ intervals along the arm and converted to an approximate arm volume using the truncated cone formula to enable estimation of volume. The presence of lymphedema was assessed by inter-limb volume difference $(>10 \%)$ based on the serial circumferential measurements in both affected and non-affected extremities. ${ }^{[19]}$ Also, the circumferential measurements of second finger base and metacarpophalangeal areas were recorded for patients with hand edema. Lymphedema was defined as an increase in hand circumference at any level by $1.5 \mathrm{~cm}$ or more compared to the contralateral side. ${ }^{[20]}$

The demographic properties (age, sex, education, and marital status, occupation, Body Mass Index) and clinical characteristics (duration of lymphedema, involvement side, treatments, type of surgery, grade of lymphedema) were recorded.

\section{Translation and cross-cultural adaptation}

After obtaining written permission from the researcher who developed the LYMQOL, the forward-backward translation method was initiated. Four steps were used in the linguistic validation of the questionnaire. Two lecturers graduated from departments of foreign languages of Bilkent University and two lymphedema specialists, competent who were fully bilingual in both Turkish and English, participated in the translation process. One of the lecturers translated the English version into Turkish to produce an understandable and conceptually equivalent translation. The back translation of the Turkish version into source language was done by the second lecturer who was blind to the purpose of 
translation. The original form and the one translated form from Turkish to English were compared by the two lecturers, and the final form of the Turkish version was prepared. Finally, the two lymphedema specialists, who were fully competent in both languages, controlled and revised the lecturers' final Turkish version to obtain the Turkish version used in this study. To avoid misunderstanding and to obtain difficulties in understanding, the instrument was given as a pretest to 30 patients with BCRL. Face-to-face interviews with the patients showed that all the indices were clear and the instrument was understandable. We made no cross-cultural validation in the translation, as feedback from the pretest study group did not identify any concerns. The Turkish version of LYMQOL-Arm was answered by the patients themselves. One lymphedema specialist was in the interview room to help the patients in case they needed assistance, which was the case only in a few patients with difficulty in reading. The scale was completed by each patient twice with one-week interval.

\section{Questionnaires}

The LYMQOL-Arm has been developed to assess the impact of lymphedema of the arms on the QoL of the patients. It consists of four domains with 28 items. These domains are symptoms, appearance, function, and mood. The answers were evaluated on a four-point Likert scale $(1=$ not at all $2=$ a little, $3=$ quite a bit, $4=\mathrm{a}$ lot). Each item received a score between 1 and 4, with higher scores indicating a worse QoL. Domain totals were calculated by adding the individual scores and dividing the total by the number of questions answered (If $>50 \%$ of questions per domain were not answered this cannot be calculated *and $=0$ ). If the item was not scored and left blank or not applicable, this was scored with a 0 . The four domains and their corresponding questions are: Function 1 (a-h), 2,3, Appearance 4,5,6,7,8 Symptoms 9,10,11,12,13,14 and Emotion 15,16,17,18,19,20. Overall QoL (Q21) is scored as the value marked by the patient, between 0-10. Previous reports indicated that the LYMQOL-Arm was easy to complete with clear face validity and good internal consistency. ${ }^{[15]}$

Patients with BCRL were also administered the Functional Assessment of Cancer Therapy-Breast-4 $(\text { FACT-B }+4)^{[21]}$ and EORTC-BR23 ${ }^{[22]}$ QoL tools, for comparisons with the new instrument. They were chosen as criterion standards, since they are suggested for QoL studies for lymphedema patients and their Turkish versions have previously been evaluated for test-retest reliability and validity. ${ }^{[10,16]}$
The European Organization for Research and Treatment ofCancerQoLBreastCancer-specific Version (EORTC QLQ-BR23) is a 23 item self-administered cancer specific questionnaire designed to measure QoL in breast cancer population at various stages and with patients with differing treatment modalities. The assessment is comprised of eight domains (body image (BRBI), sexual functioning (BRSEF), sexual enjoyment (BRSEE) and future perspective (BRFU); symptom scales-arm symptoms (BRAS), breast symptoms (BRBS), systemic therapy side effects (BRST) and upset by hair loss (BRHL). ${ }^{[23]}$ The EORTC-QLQ-BR23 is found effective and suggested in clinical studies for assessing breast cancer-specific QoL. ${ }^{[22]}$

The FACT-B +4 questionnaire was developed by adding a four-item Arm subscale to the well-validated FACT-B (Functional Assessment of Cancer TherapyBreast) ${ }^{[21]}$ and was been translated and field-tested in Turkish subjects. The 40 items in FACT-B+4 evaluated patients' physical, social, emotional and functional well-being; specific breast cancer concerns; arms symptoms; as well as their overall QOL. Previous reports indicated excellent reliability, internal consistency and sensitivity to change among patients with BCRL. ${ }^{[17,21]}$

\section{Procedure}

The participants completed the LYMQOL-Arm, FACT-B and EORTC BR23 concurrently, while the LYMQOL-Arm was completed for patients twice with seven-day intervals.

\section{Statistical analysis}

For the reliability and validity studies, the sample size is determined as at least five-fold of item numbers. Based on these data, 135 patients were required to study. A total of $20 \%$ of these patients was evaluated for retest procedure. ${ }^{[24]}$ Descriptive analyses were performed to calculate means and standard deviations and median of the demographic variables.

For test-retest reliability, 65 patients completed the questionnaires, as a second time within seven days, at the same time of day, during a non-treatment period. Intra-class correlation coefficients (ICC) with oneway random effects model, were used to determine test-retest reliability of the scores on five domains of LYMQOL and of the score on each question separately. Cronbach alpha coefficients were used to determine internal consistency of the entire questionnaire and of each domain. As recommended, the internal consistency of a magnitude of $\geq 0.70$ was sought. The Cronbach alpha was determined as high correlation, 
if values in range of 0.80 to 0.95 were obtained, where a value $>0.95$ indicated excessive internal consistency.

Confirmatory factor analysis was used to show the convenience of factor structure to the original scale. Construct validity was tested by convergent-divergent validity approach comparing the correlation of the similar scales of the LYMQOL-Arm and EORTC-BR23 and FACT-B4. It was expected that conceptually related scales would correlate better with the functioning scales of the LYMQOL and vice versa. We used the Pearson correlation coefficient for normally distributed scores and the Spearman correlation coefficient for the other scores. The correlation coefficients are interpreted as follows: $<0.4$ was weak, $0.4-0.74$ was moderate,
0.75 to 0.9 was strong, and $>0.9$ was very strong. ${ }^{[24]}$ Statistical analysis was performed using the SPSS version 15.0 statistical software (SPSS Inc., Chicago, IL, USA). A $p$ value of $<0.05$ was considered statistically significant.

\section{RESULTS}

Of 135 patients with BCRL, 65 completed test 1 and 2 for LYMQOL-Arm for test-retest analyses. The time between test 1 and 2 was within seven days. The demographic and clinical characteristics of the patients are shown in Table 1. All patients underwent breast cancer surgery. A great number of them were married and housewives. A number of participants (65\%) were

Table 1. Demographic and clinical characteristics of patients $(n=135)$

\begin{tabular}{|c|c|c|c|c|}
\hline & $\mathrm{n}$ & Mean \pm SD & Median & Min-Max \\
\hline Age (year) & & $51.8 \pm 9.8$ & & $31-82$ \\
\hline \multicolumn{5}{|l|}{ Marital status } \\
\hline Unmarried & 18 & & & \\
\hline Married & 110 & & & \\
\hline Divorced/widowed & 7 & & & \\
\hline \multicolumn{5}{|l|}{ Education } \\
\hline Primary school & 49 & & & \\
\hline Secondary school & 15 & & & \\
\hline Lycee & 36 & & & \\
\hline University & 35 & & & \\
\hline \multicolumn{5}{|l|}{ Occupation } \\
\hline Housewife & 86 & & & \\
\hline Retired & 21 & & & \\
\hline Officer & 23 & & & \\
\hline Self-employer & 5 & & & \\
\hline \multicolumn{5}{|l|}{ Involved side } \\
\hline Right & 70 & & & \\
\hline Left & 65 & & & \\
\hline Body Mass Index $\left(\mathrm{kg} / \mathrm{m}^{2}\right)$ & & $29.2 \pm 5.4$ & & $19.2-45.1$ \\
\hline Duration of lymphedema (months) & & $21.1 \pm 38.7$ & 6 & $0.2-164$ \\
\hline \multicolumn{5}{|l|}{ Dominant hand } \\
\hline Right & 128 & & & \\
\hline Left & 7 & & & \\
\hline Surgery & 135 & & & \\
\hline Modified radical mastectomy & 98 & & & \\
\hline Lumpectomy & 7 & & & \\
\hline Simple mastectomy & 30 & & & \\
\hline \multicolumn{5}{|l|}{ Lymphedema stage } \\
\hline 1 & 39 & & & \\
\hline 2 & 84 & & & \\
\hline 3 & 2 & & & \\
\hline \multicolumn{5}{|l|}{ Treatment } \\
\hline Surgery & 135 & & & \\
\hline Chemotherapy & 128 & & & \\
\hline Radiation therapy & 96 & & & \\
\hline Hormone therapy & 97 & & & \\
\hline Time since surgery (month) & & $41.4 \pm 44.5$ & & $0.75-164$ \\
\hline
\end{tabular}

SD: Standard deviation; Min: Minimum; Max: Maximum. 
Table 2. Internal consistency and test-retest reliability of the Turkish LYMQOL-Arm instrument $(\mathrm{n}=65)$

\begin{tabular}{lcccc}
\hline \multirow{2}{*}{ LYMQOL-Arm scores } & \multicolumn{2}{c}{ Test-retest } & & Consistency \\
\cline { 2 - 3 } Function & ICC & $95 \%$ CI & Cronbach alpha \\
Appearance & 0.611 & $0.373-0.773$ & 0.880 \\
Symptom & 0.665 & $0.449-0.808$ & 0.881 \\
Mood & 0.714 & $0.521-0.838$ & 0.886 \\
Overall & 0.451 & $0.165-0.666$ & 0.853 \\
\hline
\end{tabular}

LYMQOL: Lymphedema-Quality-of-Life-Questionnaire; ${ }^{*} \mathrm{p}<0.001$. ICC: Intra-class correlation-coefficient.

high school or university graduated. Radiation therapy and chemotherapy were completed prior to entry into the study. The median duration of lymphedema was six months.

\section{Reliability}

The internal consistency (based on a Cronbach alpha score of (0.85-0.90) and test-retest reliability (based on an intra-class correlation coefficient of 0.45-0.74 of the LYMQOL-Arm were found to be high $(\mathrm{p}<0.001 ;$ Table 2). The test-retest reliability of the symptom, appearance, and mood scores was strong and the function and overall scores were moderate to strong.

\section{Confirmatory factor analysis}

The confirmatory factor analysis was applied to the factor structure. Specifically, we expected a bestfit model with the following indices: a Satorra-Bentler scaled chi-square $\left(\mathrm{S}-\mathrm{B} \chi^{2}\right) /$ degrees of freedom ratio (CMIN/DF) of 2.0 or less; a Trucker Lewis Index
(TLI) of 0.90 or higher; a Comparative Fit Index (CFI) of 0.90 or higher; a Goodness-of-Fit Index (GFI) of 0.90 or higher; a Incremental Fit Index (IFI) of 0.90 or higher and a low root mean square error of approximation (RMSEA) of 0.08 or less. These values were calculated as CMIN/df: 1.733, RMSEA: 0.074, GFI: 0.782, IFI: 0.904, CFI: 0.902, TLI: 0.888. Accordingly, this factor structure was found to be acceptable.

\section{Construct (convergent-divergent) validity}

Table 3 shows an overview of inter-scale Spearman correlation coefficients between the different domains of LYMQOL-Arm and the EORTC-BR23 and FACT-B4. All patients completed the three questionnaires. All domains of LYMQOL-Arm had the strongest correlation with the expected domains of EORTC-BR23 (future, systemic complications, breast symptoms, arm symptoms). Concurrently, all subscores of LYMQOL-Arm had strongest correlations with the corresponding domains of FACT-B4. By means of divergent validity, no correlation between

Table 3. Correlation between the EORTC-BR23 and FACT-B4 questionnaires and the LYMQOL-Arm questionnaire (construct validity)

\begin{tabular}{|c|c|c|c|c|c|}
\hline & $\begin{array}{l}\text { LYMQOL-Arm } \\
\text { function }\end{array}$ & $\begin{array}{c}\text { LYMQOL-Arm } \\
\text { appearance }\end{array}$ & $\begin{array}{l}\text { LYMQOL-Arm } \\
\text { symptom }\end{array}$ & $\begin{array}{l}\text { LYMQOL-Arm } \\
\text { mood }\end{array}$ & $\begin{array}{c}\text { LYMQOL-Arm } \\
\text { overall }\end{array}$ \\
\hline FACT-B4 physical & $-0.440^{* *}$ & $-0.407^{\star *}$ & $-0.504^{* *}$ & $-0.530^{* *}$ & $0.471^{\star *}$ \\
\hline FACT-B4 social & -0.100 & $-0.173^{*}$ & -0.102 & -0.161 & $0.239^{* *}$ \\
\hline FACT-B4 emotion & $-0.356^{* *}$ & $-0.377^{\star *}$ & $-0.354^{* *}$ & $-0.512^{\star *}$ & $0.487^{\star *}$ \\
\hline FACT-B4 function & $-0.282^{* *}$ & $-0.363^{* *}$ & $-0.296^{* *}$ & $-0.363^{\star *}$ & $0.450^{* *}$ \\
\hline FACT-B4 arm symptom & $-0.318^{\star *}$ & $-0.395^{\star *}$ & $-0.371^{\star *}$ & $-0.430^{* *}$ & $0.341^{\star *}$ \\
\hline EORTC-body image & $-0.320^{\star *}$ & $-0.347^{\star *}$ & $-0.248^{\star *}$ & $-0.403^{\star *}$ & $0.370^{\star *}$ \\
\hline EORTC-sexual function & 0.026 & -0.034 & -0.022 & -0.017 & $0.192^{*}$ \\
\hline EORTC-sexual satisfaction & 0.116 & 0.086 & 0.008 & -0.064 & 0.095 \\
\hline EORTC-future & $-0.255^{\star *}$ & $-0.320^{* *}$ & $-0.214^{*}$ & $-0.406^{* *}$ & $0.203^{*}$ \\
\hline EORTC-systemic completed & $0.279^{\star \star}$ & $0.284^{\star *}$ & $0.287^{\star *}$ & $0.390^{* *}$ & $-0.335^{\star \star}$ \\
\hline EORTC-breast symptoms & $0.341^{* *}$ & $0.331^{\star *}$ & $0.373^{* *}$ & $0.429^{* *}$ & $-0.215^{\star}$ \\
\hline EORTC-arm symptoms & $0.612^{* *}$ & $0.637^{\star *}$ & $0.602^{\star *}$ & $0.561^{* *}$ & $-0.389^{* *}$ \\
\hline EORTC-hair loss & -0.092 & 0.106 & -0.096 & 0.214 & -0.102 \\
\hline
\end{tabular}


sexuality and hair loss domains of EORTC-BR23 and domains of LYMQOL-Arm (except correlation between overall score) was detected (Table 3 ).

\section{DISCUSSION}

Lymphedema research has gained acceleration recently. Breast cancer-related lymphedema is an important argument to assess and monitor different therapeutic approaches and has a significant impact on QoL. ${ }^{[3,4,8,10,16]}$ A systematic review investigated the quality and appropriateness of patient-reported outcome instruments and indicated that lymphedemaspecific QoL instruments had strong psychometric properties and offered greater reliability and validity for use in BCRL survivorship researches. ${ }^{[10]}$ As it is well-known, the perceived health status and QoL show variability in BCRL patients with different ethnic origins. In addition, factors such as socioeconomic status, as well as number and quality of medical services vary from country to country and affect the perception of QoL. ${ }^{[3,8,10,16,17]}$ Until now, there is no validated Turkish patient-reported QoL instrument for lymphedema patients. The LYMQOL is a relatively short instrument for patient-reported outcome measures that evaluates QoL in lymphedema patients and was originally developed in the UK. ${ }^{[15]}$ The transcultural validation of LYMQOL was reported previously only for Dutch patients, ${ }^{[25]}$ but was used in several studies. ${ }^{[26-29]}$

In this study, we tested the reliability and validity of LYMQOL-Arm in Turkish BCRL patients. In our study, there was a strong correlation between all items of the Turkish LYMQOL questionnaire, which demonstrates good internal consistency. We found that all subscales of Turkish LYMQOL had good internal consistency and test-re-test reliability. The Turkish version was reliable and internally consistent for the total questionnaire and subclass domains. (Cronbach alpha: 0.90). The reliability of the Turkish LYMQOL was good to excellent for patients with BCRL. Similar to van de Pas et al. ${ }^{[25]}$ who tested the validity of Dutch LYMQOL, we found Cronbach alpha coefficients higher than the recommended level of 0.70 . Our results were comparable with findings from the original validation study in UK patients with BCRL. ${ }^{[15]}$ In the reliability analysis of the present study, the Cronbach alpha values of all subscales of LYMQOL-Arm were satisfactory which were consistent with other validation studies. ${ }^{[1-14,25]}$

The test re-test correlation coefficient measured by ICC showed the stability of LYMQOL-Arm in the Turkish sample. Our results were quite similar to those reported by van de Pas ${ }^{[24]}$ who found test-retest reliability to be excellent and internal consistency to be good. In the original study, Keeley et al. ${ }^{[15]}$ showed an internal consistency using Cronbach alpha scores ranging from 0.83 to 0.88 across all domains of the instrument in patients with upper limb lymphedema. Better than the original study internal consistency in this present study was observed to be between $0.85-0.90$ which can be explained by the use of more specific breast-cancer QoL questionnaire (EORTC-BR23) rather than EORTC-30, as suggested in previous studies. ${ }^{[22]}$

In the present study, we used EORTC-QLQ-BR23 and FACT-B+4 for validity analysis. The EORTC QLQ-BR23 is a health-related QoL instrument that has been designed for patients with different stages of breast-cancer. ${ }^{[23]}$ The FACT-B+4 was recommended by the Breast Cancer Edge Task force to assess QoL in patients with BCRL. ${ }^{[21]}$ The Turkish LYMQOL-Arm showed significant correlation with FACT-B +4 and EORTC-BR23. Construct validity was tested in two ways and provided good results in our patient group. There was a good convergence between functional, symptom, appearance, mood scales of LYMQOL-Arm and domains of FACT-B+4 and EORTC-BR23. Symptom, function appearance and mood domains of LYMQOL-Arm had strong to moderate correlations with the expected domains of FACT-B+4 and EORTC-BR23. None of the domains (except overall score) of LYMQOL-Arm correlated with sexuality and hair loss scores in EORTC-BR23 questionnaire.

The results of this validity study can be compared favorably to those of other prior studies. ${ }^{[15,25,30,31]} \mathrm{In}$ the original study Spearman's correlation coefficients between the common scores of the LYMQOL and EORTC-30 ranged from- 0.68 to 0.93 were relatively close to the range of correlations found in our study. ${ }^{[15]}$ Similar to some previous studies, a more significant correlation was observed between the LYMQOL-Arm and FACT-B+4 scores supporting the external validity of the LYMQOL. ${ }^{[30,31]}$

By means of divergent validity, the LYMQOL scores were weakly correlated or showed no correlation with perceived sexuality and hair loss domains of EORTC-BR-23, as we predicted. The low or lack of correlation of some domains of EORTC-BR23 (sexuality and hair loss) with the LYMQOL subscale scores indicates that these are specific problems that may not affect the QoL in Turkish lymphedema patients. The relationship between breast cancer and sexual life 
in Turkish patients was investigated previously and shown that sexual life has far less importance than survival. ${ }^{[5]}$ Demirci et al. ${ }^{[7]}$ demonstrated that sexuality was not an important factor for QoL in breast cancer patients living in Turkey. These issues can be explained by several reasons such as sociocultural status of the Turkish women, body image, pain, fear of recurrence. Another explanation may be that the instruments measure different aspects, LYMQOL concerns the lymphedema, others the QoL-related breast cancer and treatments. ${ }^{[5-7,15,21,32]}$

There is an unmet need for a specific instrument like LYMQOL to establish patients' QoL aspect for lymphedema and to measure and monitor improvement in the treatments. Appropriate translation of lymphedema-specific QoL tools into local language to meet the needs of the community is important. To the best of our knowledge, our study is the first to investigate the Turkish validity of a specific QoL measure for lymphedema patients in Turkey. Nonetheless, the study may have potential limitations. First, it was a cross-sectional study with the lack of responsiveness data. Therefore, further studies are needed for responsiveness of this tool. Another limitation was the exclusion of patients with psychiatric diagnoses and elderly. These patients were excluded based on the perception that they were unable to have a full comprehension of all questions. These selection criteria might have caused some limitations in the generalizability of our results for all BCRL patients.

In conclusion, the Turkish translated version of LYMQOL-Arm is a valid and reliable tool for the assessment of the QoL in patients with BCRL. The Turkish BCRL patients found the Turkish version of LYMQOL-Arm questionnaire to be easy to understand. Based on our study results, we recommend the use of LYMQOL-Arm in Turkish BCRL patients in further clinical researches and suggest the investigation of the responsiveness of this questionnaire.

\section{Acknowledgements}

The authors thank to Dr. İpek Genç and Bahar Tezer Alanya for their effort during backward and forward translation processes.

\section{Declaration of conflicting interests}

The authors declared no conflicts of interest with respect to the authorship and/or publication of this article.

\section{Funding}

The authors received no financial support for the research and/or authorship of this article.

\section{REFERENCES}

1. Eser S, Yakut C, Özdemir R, Karakilinç H, Özalan S, Marshall SF, et al. Cancer incidence rates in Turkey in 2006: a detailed registry based estimation. Asian Pac J Cancer Prev 2010;11:1731-9.

2. Taghian NR, Miller CL, Jammallo LS, O’Toole J, Skolny MN. Lymphedema following breast cancer treatment and impact on quality of life: a review. Crit Rev Oncol Hematol 2014;92:227-34.

3. Paskett ED, Dean JA, Oliveri JM, Harrop JP. Cancer-related lymphedema risk factors, diagnosis, treatment, and impact: a review. J Clin Oncol 2012;30:3726-33.

4. Cancer topics: Breast cancer survival.2013; https://seer. cancer.gov/statfacts/html/breast.html.survival [Accessed: June 5, 2013]

5. Alicikus ZA, Gorken IB, Sen RC, Kentli S, Kinay M, Alanyali $\mathrm{H}$, et al. Psychosexual and body image aspects of quality of life in Turkish breast cancer patients: a comparison of breast conserving treatment and mastectomy. Tumori 2009;95:212-8.

6. Kibar S, Dalyan Aras M, Ünsal Delialioğlu S. The risk factors and prevalence of upper extremity impairments and an analysis of effects of lymphoedema and other impairments on the quality of life of breast cancer patients. Eur J Cancer Care (Engl) 20171;26.

7. Demirci S, Eser E, Ozsaran Z, Tankisi D, Aras AB, Ozaydemir G, et al. Validation of the Turkish versions of EORTC QLQ-C30 and BR23 modules in breast cancer patients. Asian Pac J Cancer Prev 2011;12:1283-7.

8. Davies C, Ryans K, Levenhagen K, Perdomo M. Quality of life and functional outcome measures for secondary lymphedema in breast cancer survivors. Rehabil Oncol 2014;32:7-13.

9. Mitchell K, Gleeson PB, Smith DS. Assessing quality of life for females with upper extremity lymphedema. J Womens Health Phys Ther 2008;32:7-13.

10. Pusic AL, Cemal Y, Albornoz C, Klassen A, Cano S, Sulimanoff I, et al. Quality of life among breast cancer patients with lymphedema: a systematic review of patientreported outcome instruments and outcomes. J Cancer Surviv 2013;7:83-92.

11. Viehoff PB, van Genderen FR, Wittink H. Upper limb lymphedema 27 (ULL27): Dutch translation and validation of an illness-specific health-related quality of life questionnaire for patients with upper limb lymphedema. Lymphology 2008;41:131-8.

12. Klernäs P, Johnsson A, Horstmann V, Kristjanson LJ, Johansson K. Lymphedema Quality of Life Inventory (LyQLI)-Development and investigation of validity and reliability. Qual Life Res 2015;24:427-39.

13. Devoogdt N, Van Kampen M, Geraerts I, Coremans T, Christiaens MR. Lymphoedema Functioning, Disability and Health questionnaire (Lymph-ICF): reliability and validity. Phys Ther 2011;91:944-57.

14. Weiss J, Daniel T. Validation of the lymphedema life impact scale (LLIS): a condition-specific measurement tool for persons with lymphedema. Lymphology 2015;48:128-38. 
15. Keeley V, Crooks S, Locke J, Veigas D, Riches K, et al. A quality of life measure for limb lymphedema (LYMQOL). J Lymphoedema 2012;5:26-37.

16. Filazoglu G, Griva K. Coping and social support and health related quality of life in women with breast cancer in Turkey. Psychol Health Med 2008;13:559-73.

17. Kaya T, Karatepe AG, Günaydn R, Yetiş H, Uslu A. Disability and health-related quality of life after breast cancer surgery: relation to impairments. South Med J 2010;103:37-41.

18. International Society of Lymphology. The diagnosis and treatment of peripheral lymphedema: 2013 Consensus Document of the International Society of Lymphology. Lymphology 2013;46:1-11.

19. Karges JR, Mark BE, Stikeleather SJ, Worrell TW. Concurrent validity of upper-extremity volume estimates: comparison of calculated volume derived from girth measurements and water displacement volume. Phys Ther 2003;83:134-45.

20. Moseley A, Piller N. The assessment and care of the patient with secondary limb lymphoedema. Aust Nurs J 2002;10(2):suppl1-4.

21. Brady MJ, Cella DF, Mo F, Bonomi AE, Tulsky DS, Lloyd SR, et al. Reliability and validity of the Functional Assessment of Cancer Therapy-Breast quality-of-life instrument. J Clin Oncol 1997;15:974-86.

22. Nguyen J, Popovic M, Chow E, Cella D, Beaumont JL, Chu D, et al. EORTC QLQ-BR23 and FACT-B for the assessment of quality of life in patients with breast cancer: a literature review. J Comp Eff Res 2015;4:157-66.

23. Sprangers MA, Groenvold M, Arraras JI, Franklin J, te Velde A, Muller M, et al. The European Organization for Research and Treatment of Cancer breast cancer-specific quality-of-life questionnaire module: first results from a three-country field study. J Clin Oncol 1996;14:2756-68.

24. Koo TK, Li MY. A Guideline of Selecting and Reporting Intraclass Correlation Coefficients for Reliability Research. J Chiropr Med 2016;15:155-63.
25. van de Pas CB, Biemans AA, Boonen RS, Viehoff PB, Neumann HA. Validation of the Lymphoedema Quality-ofLife Questionnaire (LYMQOL) in Dutch Patients Diagnosed with Lymphoedema of the Lower Limbs. Phlebology 2016;31:257-63.

26. Lee TS, Morris CM, Czerniec SA, Mangion AJ. Does Lymphedema Severity Affect Quality of Life? Simple Question. Challenging Answers. Lymphat Res Biol 2018;16:85-91.

27. Iuchi T, Dai M, Sanada H, Okuwa M, Nakatani T, Sugama J. Associations between the treatments and outcomes of patients with upper and lower lymphoedema in Japan: a cross-sectional observational study. Int J Nurs Stud 2015;52:913-9.

28. Gratzon A, Schultz J, Secrest K, Lee K, Feiner J, Klein RD. Clinical and Psychosocial Outcomes of Vascularized Lymph Node Transfer for the Treatment of Upper Extremity Lymphedema After Breast Cancer Therapy. Ann Surg Oncol 2017;24:1475-1481.

29. Mestre S, Calais C, Gaillard G, Nou M, Pasqualini M, Ben Amor $\mathrm{C}$, et al. Interest of an auto-adjustable nighttime compression sleeve (MOBIDERM ${ }^{\odot}$ Autofit) in maintenance phase of upper limb lymphedema: the MARILYN pilot RCT. Support Care Cancer 2017;25:2455-2462.

30. Belmonte Martínez R, Garin Boronat O, Segura Badía M, Sanz Latiesas J, Marco Navarro E, Ferrer Fores M. Functional Assessment of Cancer Therapy Questionnaire for Breast Cancer (FACT-B+4). Spanish version validation. Med Clin (Barc) 2011;137:685-8. [Abstract]

31. Bulley C, Coutts F, Blyth C, Jack W, Chetty U, Barber M, et al. A Morbidity Screening Tool for identifying fatigue, pain, upper limb dysfunction and lymphedema after breast cancer treatment: a validity study. Eur J Oncol Nurs 2014;18:218-27.

32. Pinto M, Gimigliano F, Tatangelo F, Megna M, Izzo F, Gimigliano R, et al. Upper limb function and quality of life in breast cancer related lymphedema: a cross-sectional study. Eur J Phys Rehabil Med 2013;49:665-73. 